Case Report

\title{
Floating Thumb with Simultaneous Carpometacarpal and Metacarpo-phalangeal Dislocation: A Case Report
}

\author{
Xiang Li ${ }^{1}$, Frankie Leung ${ }^{1,2}$, Christian Fang ${ }^{1,2}$, Tak Man Wong ${ }^{1,2, ~ * ~}$ \\ ${ }^{1}$ Shenzhen Key Laboratory for Innovative Technology in Orthopaedic Trauma, Department of Orthopaedics and Traumatology, The University \\ of Hong Kong-Shenzhen Hospital, Shenzhen, China \\ ${ }^{2}$ Department of Orthopaedics and Traumatology, The University of Hong Kong, Queen Mary Hospital, Pokfulam, Hong Kong \\ Email address: \\ lix7@hku-szh.org (Xiang Li), klleunga@hku.hk (F. Leung), fangcx@gmail.com (C.Fang), wongtm@hku.hk (Tak Man Wong) \\ ${ }^{*}$ Corresponding author
}

\section{To cite this article:}

Xiang Li, Frankie Leung, Christian Fang, Tak Man Wong. Floating Thumb with Simultaneous Carpometacarpal and Metacarpo-phalangeal Dislocation: A Case Report. Journal of Surgery. Vol. 7, No. 1, 2019, pp. 24-27. doi: 10.11648/j.js.20190701.15

Received: February 17, 2019; Accepted: April 2, 2019; Published: April 22, 2019

\begin{abstract}
Double dislocation of carpometacarpal and metacarpo-phalangeal joints of thumb is also called 'floating thumb metacarpal'. This rare injury usually presents with combined joints dislocation and collateral ligaments rupture, which may be predisposing to joint instability after closed reduction. We report a case of traumatic and chronic dislocation of carpometacarpal and metacarpo-phalangeal joints of left thumb in a 63-year-old housewife. Failure of non-operative treatment was found up to 1 month after injury due to missed follow-up. Closed reduction was tried but failed because of joints stiffness. Open reduction and $\mathrm{K}$-wire fixation were performed and radial collateral ligaments were repaired. Patient restored good functional outcome with no evidence of arthritis or subluxation at 1 year. Open reduction and fixation show good result in our case with delayed surgical intervention. The management of a floating thumb could be non-operative by close reduction and cast immobilization. However, closed monitoring and regular follow-up are critical after non-operative treatment to avoid potential risk of post-operative complications.
\end{abstract}

Keywords: Double Dislocation of Carpometacarpal and Metacarpo-Phalangeal, Floating Thumb, Carpometacarpal, Metacarpo-Phalangeal

\section{Introduction}

'Floating thumb' is a special term describing simultaneous carpometacarpal (CMC) and metacarpo-phalangeal (MCP) dislocation [1-3]. This rare injury was reported by some authors to treat with conservatively closed reduction and casting $[4,5]$ or percutaneous $\mathrm{K}$ wiring with open ligament repair $[1,6,7]$. To our knowledge, no Chinese patient was reported before. We report a rare case of chronic dislocation of both carpometacarpal and metacarpo-phalangeal joints of left thumb in which open reduction and k-wire fixation was performed and eventually good functional outcome was achieved.

\section{Case Presentation}

A 63-year-old Chinese woman was admitted in our hospital because of left thumb pain and reduced range of motion 1 month after injury. She had a history of left hand injury after a fall onto an outstretched hand with the wrist in dorsiflexion about 1 month ago. She was treated by closed reduction and casting in local hospital, but was found loss of reduction during follow-up.

On examination, deformity of left thumb was observed. Clinically both $\mathrm{CMC}$ and MCP were dislocated and tender. There was no neurovascular deficit and the active movement of the thumb was limited. Dislocation of combined CMC and MCP joints of first metacarpal was revealed on the X-ray (Figure 1). 


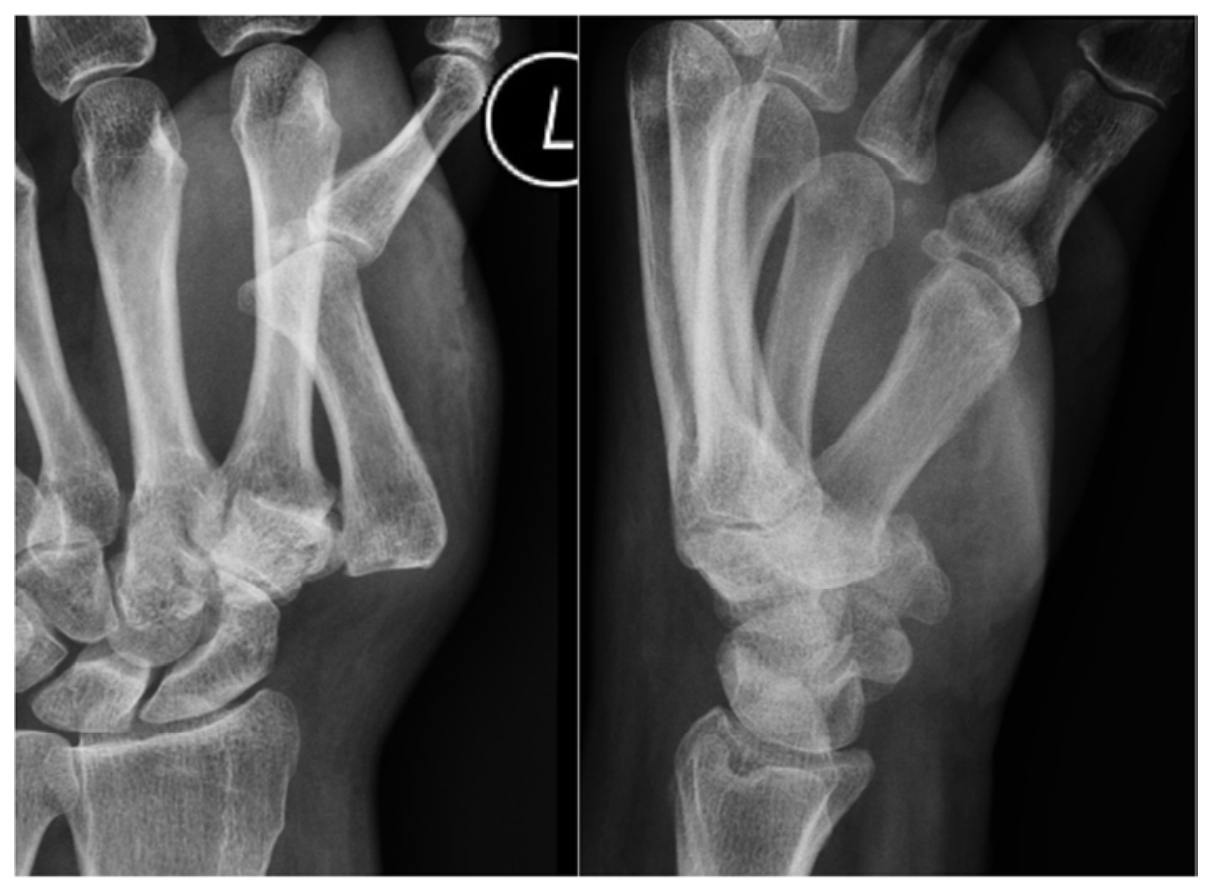

Figure 1. Anteroposterior and lateral radiographs of left thumb showed double dislocation of carpometacarpal and metacarpophalangeal joints.

In view of the chronicity of dislocation, open reduction and $\mathrm{k}$-wire was performed after a trial of closed reduction. A $3 \mathrm{~cm}$ longitudinal skin incision was made on the radial side of carpo-metacarpal joint of the left thumb. Reduction was achieved by manipulation and a $1.8 \mathrm{~mm} \mathrm{~K}$-wire was used passing from the metacarpal into the trapezoid bone for stabilization. Another $2 \mathrm{~cm}$ incision was made on the radial side of the first metacarpo-phalangeal joint to reduce the dislocation and $\mathrm{MCP}$ joint was fixed with a $1.8 \mathrm{~mm} \mathrm{~K}$-wire. Joint capsules and radial collateral ligaments were repaired with sutures. The reduction and stability were checked under image intensifier. The hand was immobilized in a thumb cast (Figure 2). The wounds and pin tracts were checked for any infection regularly. X-rays were taken to check the stability of reduction at 2 weeks post-operatively.

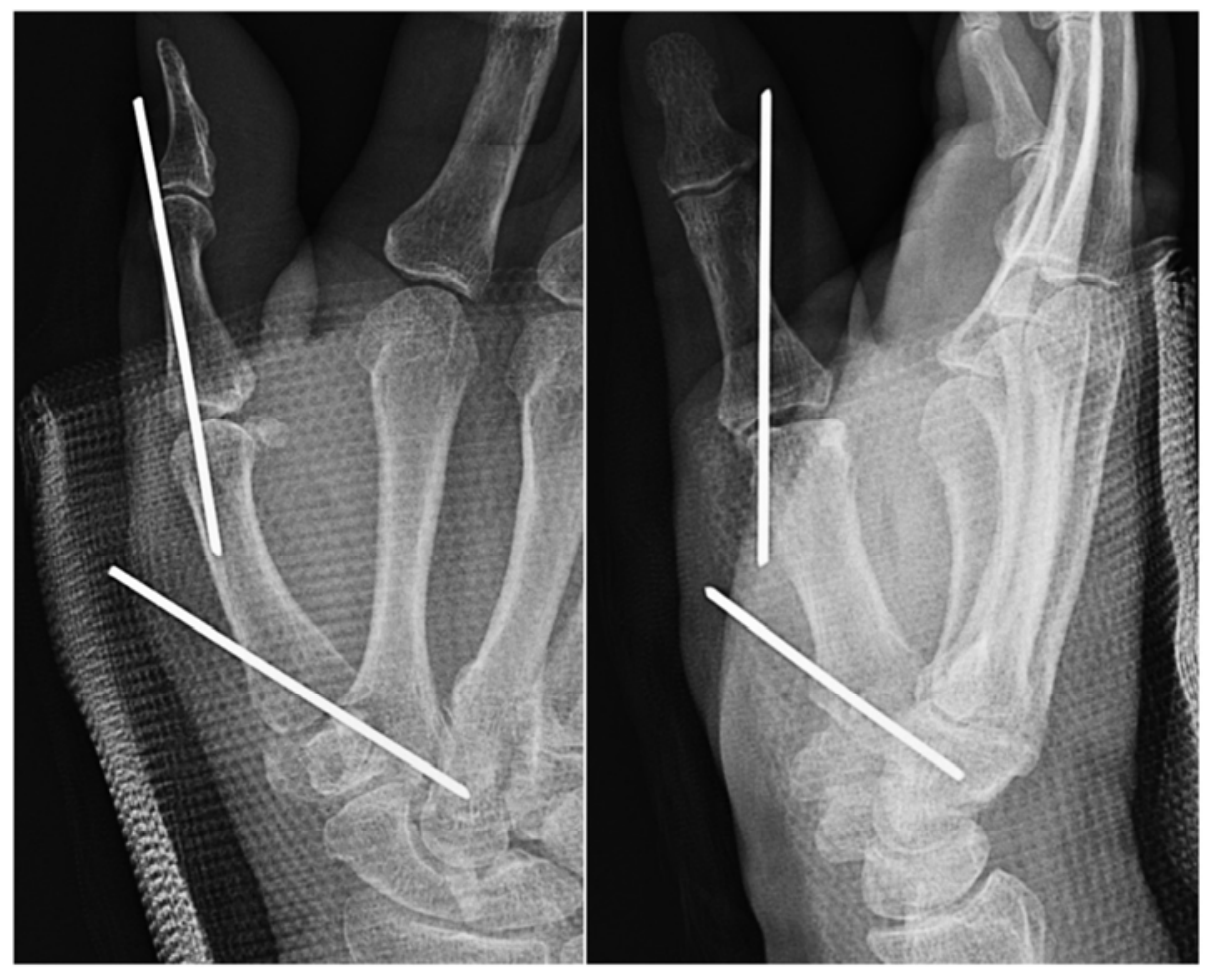

Figure 2. Open reduction and K-wire fixation of left floating thumb. 
After 6 weeks the patient was followed up in clinic and $\mathrm{X}$-ray showed both joints were well reduced. K-wires and cast were removed, and the patient was referred to occupational therapy for hand function rehabilitation.

She was assessed in the clinic at 6 months and 1 year after surgery. She regained pain free motion of the thumb but felt slightly painful after prolonged housework. Quick-DASH score was 15.9 at 6 months and 9.1 at 1 year respectively. Kapandji score was 5 at 6 month and 7 at 1 year respectively. At 1 year follow-up, the range of motion of both CMC and MCP was satisfactory as shown in Table 1 . There was no evidence of joint instability or post-traumatic arthritis seen on $\mathrm{X}$-ray at 1 year (Figure 3 ).

Table 1. Range of motion for CMC and MCP joints of the thumbs at 1 year post-operatively.

\begin{tabular}{|c|c|c|c|}
\hline \multicolumn{4}{|c|}{ Range of motion } \\
\hline & & Right & Left \\
\hline \multirow[t]{2}{*}{$\mathrm{CMC}$} & Palmar adduction/abduction & Contact/52 & Contact $/ 51$ \\
\hline & Radial adduction/abduction & Contact $/ 60$ & Contact/59 \\
\hline \multirow[t]{2}{*}{$\mathrm{MCP}$} & Extension & 25 & 0 \\
\hline & Flexion & 42 & 41 \\
\hline
\end{tabular}

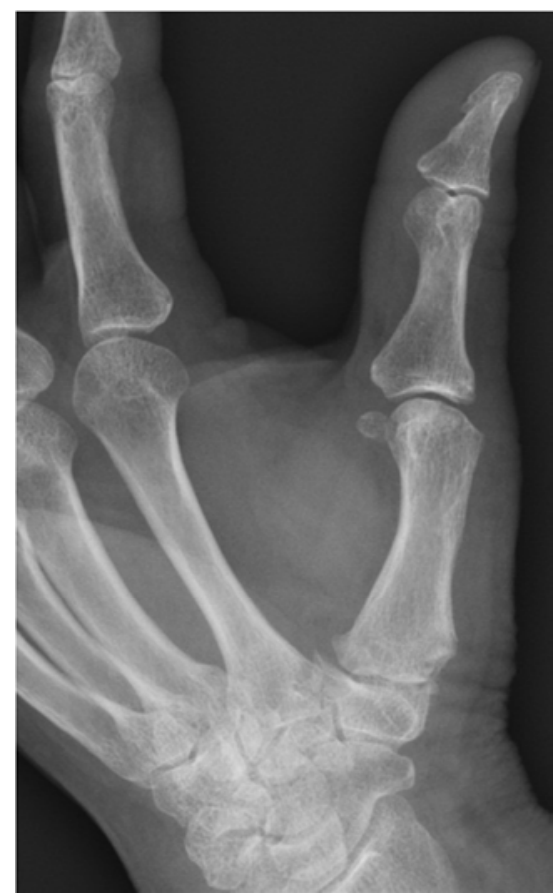

Figure 3. Radiograph of the left thumb at 1 year post-operatively.

\section{Discussion}

Double dislocation of CMC and MCP joints of the thumb is a rare injury, which is also called 'floating thumb'. Since it was first described by Moore et al. in 1978, only a few cases with simultaneous double thumb dislocation were reported [1, $5-10]$, in which most patients were almost aged $20 \mathrm{~s}$ or $30 \mathrm{~s}$ when they got injured. To our knowledge, this was the first case that a Chinese elderly woman suffered from double dislocation of CMC and MCP joints.

The mechanism of floating thumb was described as a longitudinal directed force passing through the flexed MCP joint [7]. Of note, most of the reported cases were due to high-energy injury such as road traffic accident or falling from height and only one case was caused by a fall during military training [1]. However, the present case was because of slip and fall injury which is relatively a low-energy injury. This may be accounted for the patient's advanced age and overweight with the BMI of 28.6.

Although the management of a floating thumb could be non-operative by close reduction and cast immobilization [5, 11], most of the authors chose K-wire fixation to avoid loss of reduction [1, 6-8]. In our case, the patient failed in cast immobilization after closed reduction previously, which may indicate considerable instability. Besides, we tried to reduce the thumb manually but failed due to joint stiffness. Thus, open reduction was done and K-wire fixation was used to avoid recurrent dislocation. The collateral ligaments are important for the stability of MCP joint after double dislocations [12] and its rupture may be attributed to the failure of primary reduction. In this case, we also repaired the collateral ligaments to enhance the stability of the joints.

Khan et al.[9] reported a case that failed after closed reduction and received surgical treatment up to 4 weeks after injury. The post-operative assessment showed good functional recovery eventually. Our patient was firstly treated in local hospital by closed reduction and cast immobilization, but eventually the treatment failed and needed open reduction and fixation with K-wires at nearly 1 month after injury. The final surgical outcome was satisfactory which is comparable to the above literature. The patient could resume her duty as a housewife with almost equal range of motion of CMC and CMP joints compared to the normal side.

\section{Conclusion}

Double dislocation of CMC and MCP joints of the thumb is a rare injury. Only several cases were reported in the literature which advised that either non-operative or surgical fixation was feasible depending on the stability of the reduced joints and the duration of injury. Although in our case, delayed surgical treatment up to 1 month didn't turn out to be poor functional outcome, closed monitoring and follow-up should be warranted to avoid potential risk of post-operative complications.

\section{Acknowledgements}

This work was financially supported by Shenzhen Science and Technology Funding (JCYJ20170413162540673).

\section{References}

[1] Drosos, G., E. Kayias, and K. Tsioros, "Floating thumb metacarpal" or complete dislocation of the thumb metacarpal: a case report and review of the literature. Injury, 2004. 35(5): p. 545-8. 
[2] KS, S., et al., Simultaneous dislocation of the carpometacarpal and metacarpophalangeal joints of the thumb in a motorcyclist. Journal of the Formosan Medical Association = Taiwan yi zhi, 2006. 105(8): p. 670-3.

[3] Couceiro, J., et al., Thumb carpometacarpal dislocation and open dorsal metacarpophalangeal instability: a variation of the floating thumb. Arch Plast Surg, 2014. 41(2): p. 190-2.

[4] HR, T., et al., Traumatic Floating 1st Metacarpal in a 14-Year-Old Boy Managed by Close Reduction and Thumb Spica Immobilization: A Rare Case Report. 2014. 4(4): p. 44-7.

[5] Messaoudi, T., et al., Floating thumb metacarpal in a motorcyclist: A case report. Chirurgie de la main, 2015. 34(2): p. 91-93.

[6] Ibrahim, S. and M. Noor, Simultaneous dislocations of the carpometacarpal and metacarpophalangeal joints of the thumb. Injury, 1993. 24(5): p. 343-4.

[7] Rajeev, A., S. Noureldin, and D. Graham, Floating thumb with double dislocation of carpometacarpal and metacarpo-phalangeal joints. Int J Surg Case Rep, 2014. 5(12): p. 1102-5.
[8] Gerard, F., Y. Tropet, and L. Obert, Trapezo-metacarpal and metacarpo-phalangeal dislocation of the thumb associated with a carpo-metacarpal dislocation of the four fingers. Chir Main, 1999. 18(3): p. 205-8.

[9] Khan, H., P. Darcy, and P. Magnussen, Simultaneous Volar Dislocations of Carpometacarpal and Metacarpophalangeal Joints of the Thumb. J Orthop Case Rep, 2012. 2(3): p. 8-11.

[10] Moore, J., C. Webb, and R. Thompson, A complete dislocation of the thumb metacarpal. J Hand Surg Am, 1978. 3(6): p. 547-9.

[11] Marcotte, A. and M. Trzeciak, Nonoperative treatment for a double dislocation of the thumb metacarpal: a case report. Arch Orthop Trauma Surg, 2008. 128(3): p. 281-4.

[12] Sakuma, M. and G. Inoue, Simultaneous dorsal dislocation of the metacarpophalangeal and carpometacarpal joints of a finger. Arch Orthop Trauma Surg, 1998. 117(4-5): p. 286-7. 